\title{
Extreme Ultraviolet Emission from Neutron Stars
}

\author{
R. S. FOSTER, ${ }^{1}$ J. EDELSTEIN, ${ }^{2}$ AND S. BOWYER ${ }^{2}$ \\ ${ }^{1}$ Remote Sensing Division, Code 7210, Naval Research Laboratory, \\ Washington, DC 20375, USA \\ ${ }^{2}$ Center for EUV Astrophysics, 2150 Kittredge Street, \\ University of California, Berkeley, CA 94720-5030, USA
}

\begin{abstract}
We summarize the detections of extreme ultraviolet (EUV) emission from neutron stars. Three firm detections have been made of spin-powered pulsars: the aged millisecond pulsar PSR J0437-4715, the middle-aged X-ray pulsar Geminga, and the radio pulsar PSR B0656+14. These observations allow us to evaluate both power-law and thermal-law emission models as the source of the EUV flux. For the case of PSR B0656+14 the lack of flux modulation with pulse period argues that the EUV radiation originates from the cooling neutron star surface rather than from a hot polar cap. If the emission is from a thermalized neutron star surface, then limits can be placed on the surface temperature. For the case of Geminga we can explain the observed EUV flux using thermal models that are consistent with standard neutron cooling scenarios. We also have a weak indication that the EUV emission from Geminga is pulsed in a manner consistent with the lowest energy channel observed with Rosat. For the case of the millisecond pulsar PSR J0437-4715 standard neutron star cooling models require surface re-heating. We compare different heating models to the data on this object. We rule out re-heating by crust-core friction, and find that models for the accretion from the interstellar medium, accretion from the white dwarf companion and a particle-wind nebula do not account for the EUV luminosity. Models of pulsar re-heating by magnetic monopole catalysis of nucleon decay are used to establish new limits to the flux of monopoles in the Galaxy. A single powerlaw source with properties derived from X-ray data cannot explain the EUV flux from PSR J0437-4715. The strongest model for explaining the EUV emission consists of a large $\sim 3 \mathrm{~km}^{2}$ polar cap heated from particle production in the pulsar magnetic field. We consider the prospects for detecting other neutron stars in the extreme ultraviolet.
\end{abstract}

\section{Introduction}

The detection of extreme ultraviolet (EUV) emission from neutron stars using the Extreme Ultraviolet Explorer ( $E U V E$ ) represents a challenging observation that requires both long integration times on the sources and a population of nearby neutron stars. Most neutron stars that we know about in the Galaxy are at distances between 0.5 and $10 \mathrm{kpc}$ away. The closest known radio pulsar is $\sim 100 \mathrm{pc}$ distant. High column densities of neutral hydrogen, particularly in the plane of the Galaxy, introduce a large photoelectric absorption in the $E U V E$ instrumental bandpass that strongly attenuates any EUV emission. Theoretical models for neutron star cooling predict that neutron stars with ages exceeding $\sim 10^{6.5}$ years will have very low or non-existent fluxes in the EUV or soft X-ray from thermal surface emission as they will have surface temperatures well below $10^{5} \mathrm{~K}$. The detection of any neutron stars as EUV sources is certainly a surprise.

As of March 1995 there are at least 706 known radio pulsars (Taylor et al. 1995) representing a $27 \%$ increase in the number of known spin-powered neutron stars since the previous published catalogue (Taylor, Manchester, \& Lyne 1993). A number of these new pulsars were found as a result of several sensitive searches for millisecond period 
TABLE 1. EUVE neutron star detections

$\begin{array}{llrlr}\text { Source } & \begin{array}{l}\text { Count } \\ \text { (ct/s) }\end{array} & \begin{array}{r}\text { Age } \\ (\mathrm{yrs})\end{array} & \begin{array}{l}\mathrm{DM} \\ \left(\mathrm{pc} \mathrm{cm}{ }^{-3}\right)\end{array} & \begin{array}{r}\text { Distance } \\ (\mathrm{pc})\end{array} \\ \text { PSR J0437-4715 } & 0.0143 & 5 \times 10^{9} & 2.65 & 140 \\ \text { PSR B0656+14 } & 0.024 & 1 \times 10^{5} & 14 & 260 \\ \text { Geminga } & 0.008 & 5 \times 10^{5} & - & <500 \\ \text { Her X-1 } & 0.18^{*} & <1 \times 10^{7} & - & <5000\end{array}$

* Lexan sky survey detection of an accretion powered source (Vrtilek et al. 1994).

pulsars at high Galactic latitudes. One of these recently discovered millisecond pulsars is PSR J0437-4715 (Johnston et al. 1993) and is located at a distance of only about $140 \mathrm{pc}$ away and is the closest known millisecond pulsar. Edelstein, Foster, \& Bowyer (1995) and Shemi (1995) argue that a number of the closest neutron stars might be EUV sources. Examination of the EUVE and Rosat databases for unidentified sources may lead to the discovery of additional pulsars from the very local population of isolated neutron stars.

\section{Observations}

Three spin-down powered pulsars have been detected with EUVE. They are the pulsars PSR B0656+14 and Geminga, plus the millisecond pulsar PSR J0437-4715. Table 1 gives the count rate, spin-down age, dispersion measure, and distance. We also list the accretion powered neutron star Hercules X-1, however this EUV source is probably not the neutron star but the system's accretion disk. The $E U V E$ sources are all coincident with the radio or X-ray source positions within the 1 arcminute uncertainty of the $E U V E$ pointing.

All three detected spin-powered pulsars were observed with the $100 \AA$ (Lexan) filter on the Deep Survey Instrument. The Geminga source has the longest integration of 250,458 seconds between 1994 January 14 to 24 . A total of 2306 counts were received in the detector which resulted in a net count of 2032 after subtracting background. The pulsar PSR B0656+14 had a shorter exposure of 91,021 seconds with 3358 photons counted during the observation. After removing the background count rate a net count of 2109 photons were detected during the observation.

The millisecond pulsar PSR J0437-4715 was observed between 1994 January 31 and February 4 for a total of $71,886 \mathrm{~s}$. The photon count rate after correcting for instrumental vignetting and telemetry losses was $0.0143 \pm 0.0008$ counts $\mathrm{s}^{-1}$. Using an assumed ionization fraction $X=N_{e} / N_{\mathrm{H}} \sim 0.1$ we can estimate the neutral hydrogen column density of $\sim 7 \times 10^{19} \mathrm{~cm}^{-2}$ from the pulsar dispersion measure of $2.65 \mathrm{pc} \mathrm{cm}^{-3}$. This source was observed by Becker \& Trumper (1993) with Rosat and shown to have pulsed $\mathrm{X}$-ray emission commensurate with the pulsars $5.75 \mathrm{~ms}$ rotational period.

The source flux for each pulsar is derived from the count rates using the Deep Survey effective area of an assumed hydrogen column $N_{\mathrm{H}}$ and a spectral model of the instrumental response. The Deep Survey response function is highly sensitive to the absorbing column densities between $10^{19} \mathrm{~cm}^{-2}$ and $10^{21} \mathrm{~cm}^{-2}$. Detection of pulse modulation was 


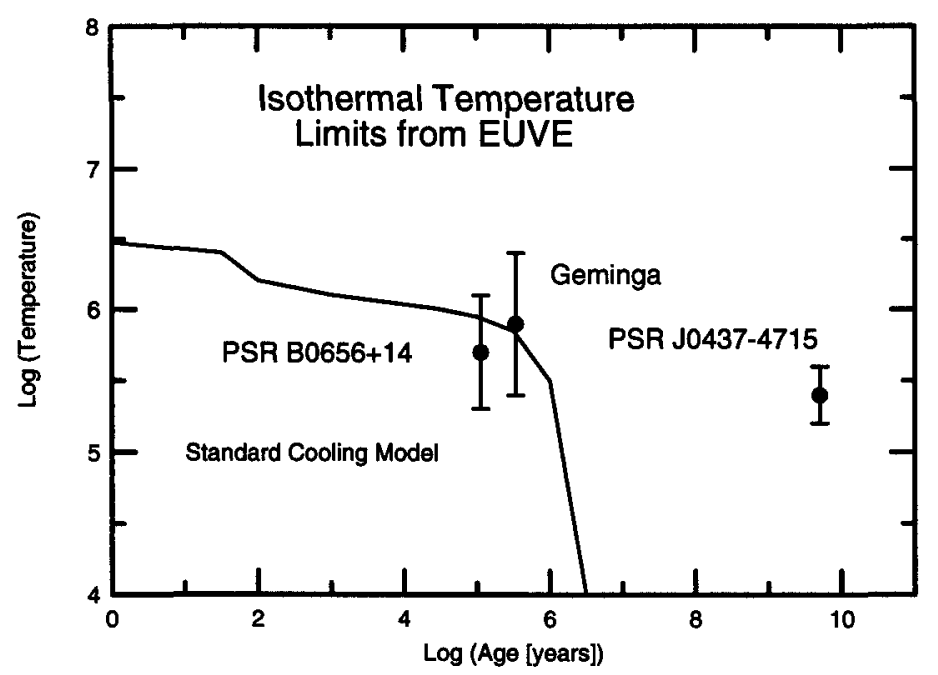

FIGURE 1. Isothermal temperate versus pulsar age for the three spin-powered pulsars detected with EUVE. Also plotted is a standard neutron star cooling model (e.g., Page \& Applegate 1992).

not possible for PSR J0437-4715 due to limited time resolution in the mode used for these observations.

\section{Discussion}

The EUVE data allow us to discriminate between different power-law models and blackbody source models for emission from nearby neutron stars. In the case of PSR J0437-4715, we found that the power-law model based upon the Rosat observations by Becker \& Trumper (1993) cannot explain the observed EUV emission. We found that the EUV data can be explained by extrapolations of the Rosat power-law model only for the case where the total absorbing column of neutral hydrogen exceeds the measured hydrogen column out of the Galaxy in this direction.

The companion star to the millisecond pulsar has been detected optically (Bell, Bailes, $\&$ Besell 1993) and identified as a cool white dwarf with a color temperature of $4000 \pm 350$ K. The star is too cool and too far ( 65 to 160 pc distant) to be the source of the EUV emission.

It is possible that the flux we are observing from PSR J0437-4715 is thermal in origin. Edelstein, Foster, \& Bowyer (1995) argued the the EUV flux and X-ray emission are both consistent with a single blackbody with a temperature of $\sim 5.7 \times 10^{5} \mathrm{~K}$ from an emitting region of $\sim 3 \mathrm{~km}^{2}$ with a Galactic absorbing column of $N_{\mathrm{H}} \sim 5 \times 10^{19} \mathrm{~cm}^{-2}$. If the emission is from the entire surface of the neutron star, then we can place a lower limit of the surface temperature of $1.6 \times 10^{5} d_{140}^{-0.5} \mathrm{~K}$ for a distance of $140 \mathrm{pc}$ and a radius of $10 \mathrm{~km}$. An upper limit constrains the temperature to be less than $4.0 \times 10^{5} d_{140}^{-0.5} \mathrm{~K}$.

Applying similar isothermal models to the observed emission from the two younger neutron stars we can derive temperature limits as a function of spin-down age. We 


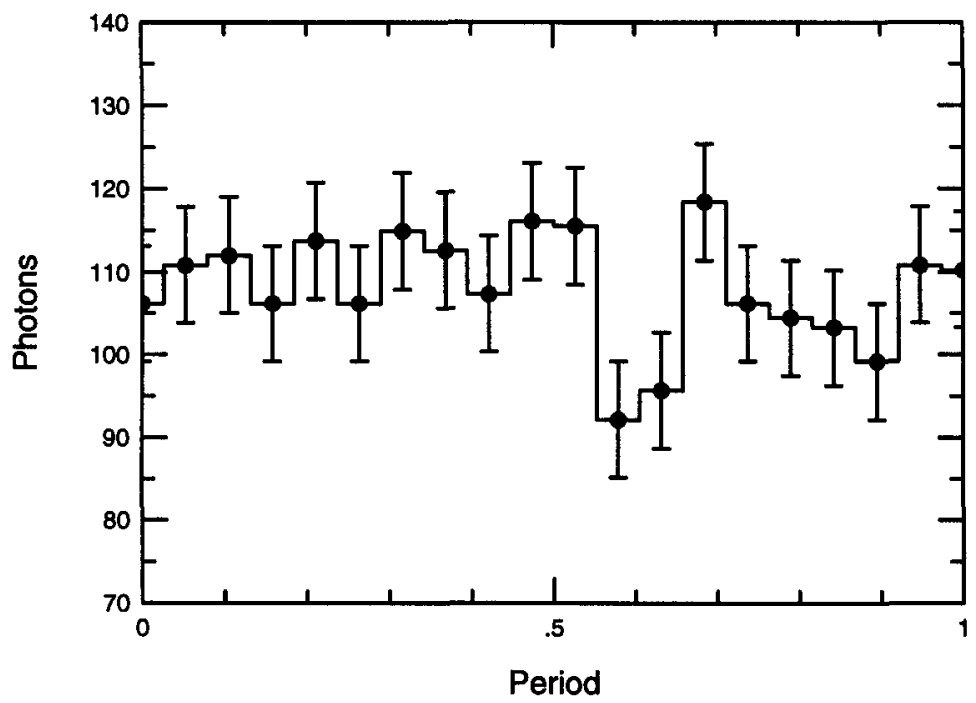

Figure 2. Extreme ultraviolet photon counts versus pulse phase for the spin-power pulsar Geminga. The pulse profile is computed using the spin-down model from the X-ray timing observations of Halpern \& Holt (1992) and Halpern \& Ruderman (1993).

plot these limits in Figure 1. Also in Figure 1 is a standard neutron star cooling curve following Page \& Applegate (1992). The two $10^{5}$ year old neutron stars fit the cooling curve while the $5 \times 10^{9}$ year old millisecond pulsar shows excess emission compared to standard cooling models. This leads us to speculate on the source of the neutron star surface re-heating that must be going on, if the EUV emission is thermal in origin.

Edelstein, Foster, \& Bowyer (1995) considered five possible mechanism for thermal reheating in old neutron stars including crust-core friction from pulsar spin-down, BondiHoyle type accretion from the ISM, accretion from the companion star, a particle-wind nebula generated from the neutron star, and magnetic monopoles. All of these suggestions are difficult to justify observationally, although the particle-wind nebula mechanism following Arons \& Tavani (1993) might explain the emission within an order of magnitude.

It has been suggested that neutron stars could be heated by magnetic "monopolecatalyzed" nucleon decay (Freese, Turner, \& Schramm 1983 and Kolb \& Turner 1984). Superheavy magnetic monopoles $\left(\leq 10^{21} \mathrm{GeV}\right)$ that hit the surface of a neutron star will lose sufficient energy through electronic interactions to be captured by the star. These monopoles accumulate inside the neutron star at a rate proportional to the monopole flux. The estimated upper limit to the Galactic magnetic monopole flux is

$$
F_{\mathrm{G}} \leq 8.3 \times 10^{-25}\left(\frac{\tau_{9}}{5}\right)^{-1}\left(\sigma_{-28} v\right)^{-1} R_{10}^{4} \beta_{-3}^{2} M_{1.4}^{-2} \mathrm{~cm}^{-2} \mathrm{sr}^{-1} \mathrm{~s}^{-1},
$$

where $\tau_{9}$ is the neutron star age in units of $10^{9} \mathrm{yr}, \sigma_{-28}$ is the interaction cross section in units of $10^{-28} \mathrm{~cm}^{2}, v$ is the relativistic velocity difference between the monopole and interacting nucleon assumed in units of the speed of light $c=3 \times 10^{10} \mathrm{~cm} \mathrm{~s}^{-1}, R_{10}$ is 
the neutron star radius in units of $10 \mathrm{~km}, \beta_{-3}$ is the monopole's velocity far from the star in units where $\beta_{-3}=10^{3} \times v c^{-1}$ (the viral velocity of a monopole in the Galaxy is $\sim 10^{-3} c$ ), and $M_{1.4}$ is the neutron star mass in solar units. The upper limit to the surface temperature of PSR 0437-4715 and the object's extreme age results in a value for $F_{\mathrm{G}}$ that is 3 orders of magnitude lower than the upper limit derived from PSR $1929+10$ (Freese, Turner, \& Schramm 1983).

The photon arrival times for the data from the millisecond pulsar PSR J0437-4715 have insufficient time resolution to identify pulsed emission. Future observations using a high time resolution mode on the EUVE spacecraft will allow for the identification of any possible pulsed emission in the photon count statistics. The photons collected from both PSR B0655+14 and Geminga were folded using the spin-down models with differing results. No pulsed emission was identified from PSR B0655+14 while a weak indication of pulsed emission exists for Geminga (see Figure 2). An inverted pulse (or dip) near the phase 0.5 is similar to the profile seen in the lowest energy $0.07-0.28 \mathrm{keV}$ band from the Rosat data (Halpern \& Holt 1992 and Halpern \& Ruderman 1993).

\section{Summary}

Three spin-powered pulsars have been detected with EUVE. All three sources have previously been detected in soft X-rays with Rosat. The two pulsars with spin-down ages less than $10^{6}$ years have EUV emission that fit standard thermal models for neutron star cooling. The apparent excess emission from the millisecond pulsar PSR 0437-4715 seen in both the extreme ultraviolet and soft $\mathrm{X}$-rays are not consistent with standard models describing thermal flux from this object. However, a large polar cap heated from the back flow of relativistic particles produced in the magnetic beam might be producing $\mathrm{a} \sim 3 \mathrm{~km}^{2}$ region that would explain both the EUV and X-ray observations below 0.4 $\mathrm{keV}$. Alternatively, if the EUV emission is due to a thermalized surface on the neutron star then we place a limit on the surface temperature of the neutron star of $1.6-4.0 \times 10^{5}$ K. Some surface re-heating mechanism would be required to explain this temperature according to the standard models for neutron star cooling.

The discovery of a number of new nearby radio pulsars and the large list of still unidentified EUV sources provides an opportunity for the detection of additional neutron stars in the EUV. Further study of this population may provide clues to the nature of the EUV emission from neutron stars.

We acknowledge useful conversations in the preparation of this work with C. Dermer, J. Arons, and M. Tavani. We also thank P. Ray and D. Chakrabarty for their assistance in providing software for the ephemeris conversions. Basic research in precision pulsar astrophysics at the Naval Research Laboratory is supported by the Office of Naval Research. The Extreme Ultraviolet Explorer is supported by NASA contract NAS5-30180.

\section{REFERENCES}

ARons, J. \& TaVaNI, M. 1993, High-energy emission from the eclipsing millisecond pulsar PSR 1957+20, ApJ, 403, 249

Becker, W. \& Trumper, J. 1993, Detection of pulsed X-rays from the binary millisecond pulsar J0437-4715, Nature, 356, 528

Edelstein, J., Foster, R. S., \& Bowyer, S. 1995, Extreme Ultraviolet Emission from the Millisecond Pulsar J0437-4715, ApJ, in press

Freese, K., TURNer, M., \& SchramM, D. N. 1983, Monopole catalysis of nucleon decay in old pulsars, Phys. Rev. L., 51, 1625 
HALPERN, J. P. \& HolT, S. S. 1992, Discovery of soft X-ray pulsations from the gamma-ray source Geminga, Nature, 357, 222

Halpern, J. P. \& Ruderman, M. 1993, Soft X-ray properties of the Geminga pulsar, ApJ, 415,286

JoHNSTON, S., ET AL. 1993, Discovery of a very bright, nearby binary millisecond pulsar, Nature, 361,613

Kolb, E. \& TURNER, M. 1984, Limits from the soft X-ray background on the temperature of old neutron stars and on the flux of superheavy magnetic monopoles, ApJ, 286, 702

PaGe, D. \& APPlEgate, J. H. 1992, The cooling of neutron stars by the direct Urca process, ApJL, 394, L17

SHEMI, A. 1995, Are Unidentified EUV Sources the Closest Neutron Stars?, MNRAS, submitted

TAYLoR, J. H., MANChESTER, R. N., \& LYNE, A. G. 1993, Catalog of 558 pulsars, ApJS, 88, 529

TAYLOR, J. H., MANChESTER, D. N., LYNE, A. G., \& CAMLO, F. 1995, in preparation

VRTIEK, S. D. ET AL. 1994, Multiwavelength observations of Hercules X-1/HZ Hercules, ApJL, 436, L9 